\title{
Role of Heme Oxygenase in Modulating Endothelial Function in Mesenteric Small Resistance Arteries of Spontaneously Hypertensive Rats
}

Enzo Porteri, Luigi F. Rodella, Rita Rezzani, Damiano Rizzoni, Silvia Paiardi, Carolina de Ciuceis, Gianluca E.M. Boari, Eleonora Foglio, Gaia Favero, Nicola Rizzardi, Caterina Platto \& Enrico Agabiti Rosei

To cite this article: Enzo Porteri, Luigi F. Rodella, Rita Rezzani, Damiano Rizzoni, Silvia Paiardi, Carolina de Ciuceis, Gianluca E.M. Boari, Eleonora Foglio, Gaia Favero, Nicola Rizzardi, Caterina Platto \& Enrico Agabiti Rosei (2009) Role of Heme Oxygenase in Modulating Endothelial Function in Mesenteric Small Resistance Arteries of Spontaneously Hypertensive Rats, Clinical and Experimental Hypertension, 31:7, 560-571, DOI: 10.3109/10641960902927978

To link to this article: http://dx.doi.org/10.3109/10641960902927978

曲 Published online: 04 Nov 2009.

Submit your article to this journal $[\pi$

Џ Article views: 49

Q View related articles ¿ 


\title{
Role of Heme Oxygenase in Modulating Endothelial Function in Mesenteric Small Resistance Arteries of Spontaneously Hypertensive Rats
}

\author{
ENZO PORTERI,${ }^{1}$ LUIGI F. RODELLA, ${ }^{2}$ RITA REZZANI, ${ }^{2}$ \\ DAMIANO RIZZONI, ${ }^{1}$ SILVIA PAIARDI, ${ }^{1}$ \\ CAROLINA DE CIUCEIS, ${ }^{1}$ GIANLUCA E.M. BOARI ${ }^{1}$ \\ ELEONORA FOGLIO, ${ }^{2}$ GAIA FAVERO ${ }^{2}$ NICOLA RIZZARDI, ${ }^{1}$ \\ CATERINA PLATTO,${ }^{1}$ AND ENRICO AGABITI ROSEI ${ }^{1}$ \\ ${ }^{1}$ Clinica Medica, Department of Medical and Surgical Sciences, University of \\ Brescia, Italy \\ ${ }^{2}$ Department of Biomedical Sciences and Biotechnology, University of Brescia, \\ Italy
}

\begin{abstract}
It has been proposed that endothelial dysfunction is due to the excessive degradation of nitric oxide (NO) by oxidative stress. The enzyme heme-oxygenase $(\mathrm{HO})$ seems to exert a protective effect on oxidative stress in the vasculature, both in animal models and in humans. The objective of this study is to evaluate the effects of inhibition or activation of $\mathrm{HO}$ on endothelial function in mesenteric small resistance arteries of spontaneously hypertensive rats (SHR). Six SHR were treated with cobalt protoporphyrin IX $50 \mathrm{mg} / \mathrm{Kg}$ (CoPP), an activator of $\mathrm{HO}$; six SHR with stannous mesoporphyrin $30 \mathrm{mg} / \mathrm{Kg}(\mathrm{SnMP})$, an inhibitor of $\mathrm{HO}$, and six SHR with saline. As controls, six Wistar-Kyoto rats (WKY) were treated with CoPP, six WKY with SnMP, and six WKY with saline. Drugs were injected in the peritoneum once a week for 2 weeks. Systolic blood pressure (SBP) was measured (tail cuff method) before and after treatment. Mesenteric small resistance arteries were mounted on a micromyograph. Endothelial function was evaluated as a cumulative concentration-response curve to acetylcholine $(\mathrm{ACH})$, before and after preincubation with $N(G)$-methyl-L-arginine (L-NMMA, inhibitor of NO synthase), and to bradykinin (BK). In SHR treatment with CoPP, improved $A C H$-and BK-induced vasodilatation (ANOVA $p<0.001)$ and this improvement was abolished by L-NMMA (ANOVA $p<0.001$ ). SnMP was devoid of effects on endothelial function. In WKY, both activation and inhibition of $\mathrm{HO}$ did not substantially affect endothelium-mediated vasodilatation. The stimulation of $\mathrm{HO}$ seems to induce an improvement of endothelial dysfunction in SHR by possibly reducing oxidative stress and increasing NO availability.
\end{abstract}

Received July 14, 2008; revised August 7, 2008; accepted August 8, 2008.

Address correspondence to Damiano Rizzoni, Department of Medical and Surgical Sciences, University of Brescia, c/o 2a Medicina Spedali Civili di Brescia, Piazza Spedali Civili 1, 25100 Brescia, Italy; E-mail: rizzoni@med.unibs.it 
Keywords vessels, small arteries, hypertension, heme oxygenase, carbon monoxide, nitric oxide, endothelial function and dysfunction

\section{Introduction}

The endothelium plays a pivotal role in transducing and modulating biochemical, humoral, and mechanical stimuli coming from the vascular lumen. It controls vascular permeability, circulating cells adhesion, and smooth muscle cells contraction and dilatation, thus modulating vascular tone.

Arterial hypertension, diabetes mellitus, dyslipidemia, and other cardiovascular risk factors may modify the cell redox state in the vessel lumen and wall and may trigger the onset of endothelial dysfunction (1-3). An increase in oxidative stress observed in these pathological conditions is driven by an excess production of reactive oxygen species, thus leading to vascular inflammation and an excessive degradation of nitric oxide. In large arteries, alterations of endothelial function promote atherosclerotic processes (4). In small arteries ( $<350 \mu \mathrm{m}$ of lumen diameter), the endothelium exerts a key role in the imbalance between vasoconstriction and vasodilatation and may induce growth responses in the tunica media of the vessels $(5,6)$. Consequently, endothelial dysfunction and damage may theoretically have an impact on vascular smooth muscle cell structure and function, intercellular matrix proteins, and, hence, on small artery remodelling, although this point is still controversial (7).

It has been demonstrated previously that the heme/heme-oxygenase system may have relevant antioxidant properties $(4,8)$. Heme oxygenase $(\mathrm{HO})$ is a cytoprotective enzyme that degrades heme (a compound with potent oxidant properties), generating carbon monoxide $(\mathrm{CO})$, a gas with vasodilator and anti-inflammatory properties, bilirubin (that is derived from biliverdin and has antioxidant effects), and iron (sequestered by ferritin). Heme oxygenase exists in two isoforms: the first one, HO-1, is an inducible enzyme that catalyzes the degradation of heme in the previously mentioned catabolites, while second, HO-2 is the constitutive form of the enzyme. Carbon monoxide and biliverdin may mediate the protective effect of HO-1 in terms of anti-inflammatory, anti-apoptotic, and antiproliferative actions (8). Carbon monoxide may stimulate soluble guanylyl-cyclase or may open calcium-activated $\mathrm{K}^{+}$channels inducing relaxation of smooth muscle cells, while biliverdin and its derivative bilirubin are potent antioxidant compounds, and may inhibit lipid peroxidation, reduce oxidative stress, and increase half-life of nitric oxide (8). On this basis, HO seems to exert a protective effect toward oxidative stress in the vasculature, both in animal models and in humans.

Spontaneously hypertensive rats (SHR) represent an animal model of genetic hypertension that from 4 to 12 weeks of age simultaneously develop high blood pressure values and endothelial dysfunction in several vascular districts, including mesenteric small resistance arteries $(9,10)$. However, no data are presently available about the effects of a pharmacological modulation of the HO system on endothelium-dependent functional responses of small resistance arteries in SHR.

Therefore, we considered it important to investigate the effects of the in-vivo inhibition or activation of $\mathrm{HO}$ on endothelial function in mesenteric small resistance arteries of SHR using a precise and reliable in-vitro ex vivo micromygraphic technique.

\section{Materials and Methods}

Thirty-six male rats were included in the study. They were $18 \mathrm{Wistar}-\mathrm{Kyoto}$ normotensive control rats (WKY) and 18 SHRs at 12 weeks of age. The animals were obtained from 
Charles River Laboratory (Calco, Italy). All the following procedures were in accordance with the institutional guidelines. The rats were housed two to a cage in a room in which the temperature was controlled from $23^{\circ} \mathrm{C}$ to $25^{\circ} \mathrm{C}$ and a 12-hour light/dark cycle was maintained. Food (standard laboratory chow) and water were supplied ad libitum. Animals were treated by injections of cobalt protoporphyrin IX (CoPP), which is an inducer of HO, at a dose of $50 \mathrm{mg} / \mathrm{Kg}$ of body weight, or stannous mesoporphyrin (SnMP), an HO inhibitor, at a dose of $30 \mathrm{mg} / \mathrm{kg}$, or saline. Treatments were injected into the peritoneum once a week for 2 weeks. The animals were subdivided in six groups (six animals per group): group 1: SHR treated with cobalt protoporphyrin IX (SHR-CoPP); group 2: SHR treated with stannous mesoporphyrin (SHR-SnMP); group 3: SHR rats treated with saline (SHR Saline); group 4: WKY treated with CoPP (WKY-CoPP); group 5: WKY treated with SnMP (WKY-SnMP); group 6: WKY treated with saline (WKY Saline). CoPP and SnMP were obtained from Sigma-Aldrich, Milan, Italy.

\section{Blood Pressure Measurement}

Systolic blood pressure (SBP) was measured noninvasively (tail cuff method, IITC Life Science Instruments, Woodland Hills, CA) in conscious rats before and after treatment. In our laboratory, the average coefficient of variation of the plethysmographic SBP measurement was $5.23 \%$ (16 rats, 10 measurements in each rat in a single session).

\section{Small Arteries Evaluation}

Seven days after the last administration of the drugs, the animals were killed by decapitation and the small intestine were dissected promptly. Four mesenteric small arteries (second-third branch) feeding the first part of ileum (about $10 \mathrm{~cm}$ from the pylorus) were isolated. The remaining part of the mesenteric tree was immediately frozen for immunohistochemical studies. At the same time, mesenteric small arteries were mounted as ring preparation on an isometric myograph (410 A, Danish Myo Technology, Aarhus, Denmark) according to Mulvany's technique $(9,10)$. The vessels segments (100 to $280 \mu \mathrm{m}$ average diameter in relaxed conditions, $2 \mathrm{~mm}$ long) were excised free of connective and adipose tissue and two stainless steel wires of $40 \mu \mathrm{m}$ diameter were treaded through the lumen. Data from four different vessels from each rat were averaged, to provide one observation per rat. Details about the micromygraphic technique of evaluation of small artery morphology were previously reported (10-13).

\section{Endothelial Function Evaluation}

Endothelial function was evaluated by testing the vasodilator responses to acetylcholine and bradykinin. We decided to explore endothelial function by using two different endothelial agonists in order to have a wider picture of endothelium-dependent responses, as previously done in similar studies $(3,14,15)$.

Vessels were precontracted with norepinephrine $10^{-5} \mathrm{~mol} / \mathrm{L}$ for 2 minutes. A concentration-response curve to acetylcholine was performed at the following cumulative concentrations: $10^{-9}, 10^{-8}, 10^{-7}, 10^{-6}, 10^{-5} \mathrm{~mol} / \mathrm{L}$. The same protocol was repeated after preincubation with $\mathrm{N}(\mathrm{G})$-methyl-L-arginine (L-NMMA, inhibitor of nitric oxide synthase) at the concentration of $300 \mu \mathrm{mol} / \mathrm{L}$. A concentration-response curve to bradykinin was also performed at the following cumulative concentrations: $10^{-10}, 10^{-9}, 10^{-8}$, $10^{-7}, 10^{-6} \mathrm{~mol} / \mathrm{L}$. 
Endothelium-independent vasodilatation was evaluated by exposing the vessels to increasing concentrations of sodium nitroprusside, after norepinephrine precontraction. The dose-response curve to sodium nitroprusside was performed at the following cumulative concentrations: $10^{-9}, 10^{-8}, 10^{-7}, 10^{-6}, 10^{-5} \mathrm{~mol} / \mathrm{L}$. Vasodilator responses were expressed as the percent decrease of the wall tension obtained with norepinephrine precontraction. HO-1 expression in mesenteric small resistance arteries was then evaluated by immunohistochemistry.

\section{HO-1 Immunohistochemistry}

After freezing, serial sections of small mesenteric arteries were cut by a cryostat (8- $\mu \mathrm{m}$ tick) and placed in gelatine-coated slides. The sections were immersed in 3\% hydrogen peroxide in methanol for $30 \mathrm{~min}$ to block the endogenous peroxidase activity. The sections were then incubated with normal goat serum (10\% in PBS containing $0.1 \%$ Triton X-100) for $60 \mathrm{~min}$, and successively with rabbit polyclonal anti-HO-1 (Stressgen, Victoria, British Columbia CANADA) diluted 1:500 in PBS, containing 3\% normal goat serum and 0.1\% Triton X-100, for 1 at room temperature and overnight at $4{ }^{\circ} \mathrm{C}$. After incubation in the primary antiserum, the sections were sequentially incubated in biotinylated goat anti-rabbit immunoglobulins and avidin-biotin peroxidase complex (Vector Labs, Burlingame, CA) and were stained by immersing the slides in a solution of $0.05 \%$ 3.3-diaminobenzidine tetrahydrochloride (DAB) (Sigma, St. Louis, MO) and $0.03 \%$ hydrogen peroxide. All slides were dehydrated and mounted by DPX (Sigma). Specificities of antibodies labelling were investigated using appropriate controls, incubating the tissue sections with non-immune rabbit serum instead of the primary antiserum.

\section{Statistical Analysis}

The results are expressed as mean \pm SD. A student's $t$-test analysis with Bonferroni's correction for multiple comparison was used for comparison of differences of means among different groups. All investigated variables were normally distributed. Statistical significance of differences among experimental groups in the vasodilator responses to acetylcholine and bradykinin was estimated using a two-way analysis of variance (ANOVA) for repeated measures. A p-value less than 0.05 was considered statistically significant.

\section{Results}

\section{Blood Pressure}

Systolic blood pressure values in SHR and WKY at baseline and after treatment (12 and 14 weeks of age) are reported in Figure 1. Treatment with CoPP and SnMP significantly decreased SBP in SHR in respect with saline $(161.8 \pm 15.7$ and $163.1 \pm 20.5 \mathrm{~mm} \mathrm{Hg}$, respectively, versus $186.1 \pm 8.5 \mathrm{mmHg}, \mathrm{p}<0.05)$. Blood pressure values after treatment compared to baseline were significantly different only in the WKY group treated with SnMP. In every case, SBP values observed in SHR, both before or after any treatment, were significantly different $(p<0.001)$ in respect to blood pressure values observed in WKY, before or after any treatment.

\section{Endothelial Function}

A significant reduction in the vasodilator responses to acetylcholine and bradykinin was observed in SHR treated with saline compared with WKY treated with saline (ANOVA 


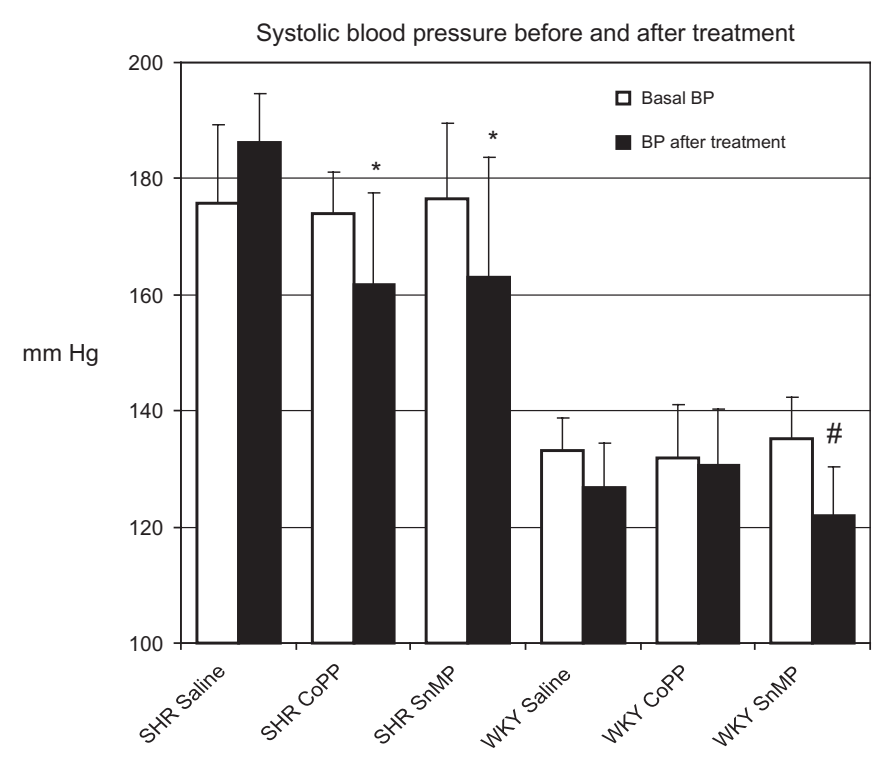

Figure 1. Systolic blood pressure (BP) values in SHR and WKY before (10 weeks of age) and after treatment (12 weeks of age) (saline, CoPP, SnMP). Values are expressed as mean \pm SD. ${ }^{*} \mathrm{p}<0.05$ vs. SHR after saline treatment; \# $\mathrm{p}<0.05$ vs. WKY SnMP at baseline (Basal), before treatment.

$\mathrm{p}<0.001$ ) (Figures 2 and 3). In SHR treated with CoPP, a significant improvement in the response to acetylcholine and bradykinin was observed (ANOVA $p<0.001$ vs. SHR treated with saline or SnMP), while SnMP was devoid of effect, when compared to saline (Figures 2 and 3). In WKY, no difference in the concentration-response curve to acetylcholine and bradykinin between saline, CoPP, or SnMP was observed. Preincubation with L-NMMA did not significantly modify the concentration-response curve to acetylcholine in SHR treated with saline and in SHR treated with SnMP, while in SHR treated with CoPP, a significant reduction in the vasodilator response was observed (ANOVA $p<0.001$ vs. no preincubation with L-NMMA; ANOVA $\mathrm{p}<0.01$ vs. WKY treated with saline after preincubation with L-NMMA, ANOVA $p=$ NS vs. SHR treated with saline after preincubation with L-NMMA), suggesting a restoration of the bioavailability of nitric oxide (NO) (Figure 2). In WKY treated with saline, CoPP or SnMP, incubation with L-NMMA slightly, but significantly reduced vasodilatation to acetylcholine (ANOVA $<0.05$ in each case).

Endothelium-independent vasodilatation (concentration-response curves to sodium nitroprusside) was not significantly different among the groups studied (data not shown).

\section{HO-1 Immunoreactivity}

Semi-quantitave analysis of HO-1 vascular content is reported in Table 1. HO-1 immunoreactivity appeared as brown spots and it was localized in the cytoplasm of endothelial and vascular smooth muscle cells. HO-1 positive cells were also found in the adventitia. In the WKY rats, we observed a moderate staining in the tunica intima, the media, and the adventitia (Figure 4). In SHR, the HO-1 staining was similar to that found in WKY (Table 1, Figure 4), although brown staining appeared more evident within the tunica media than in 


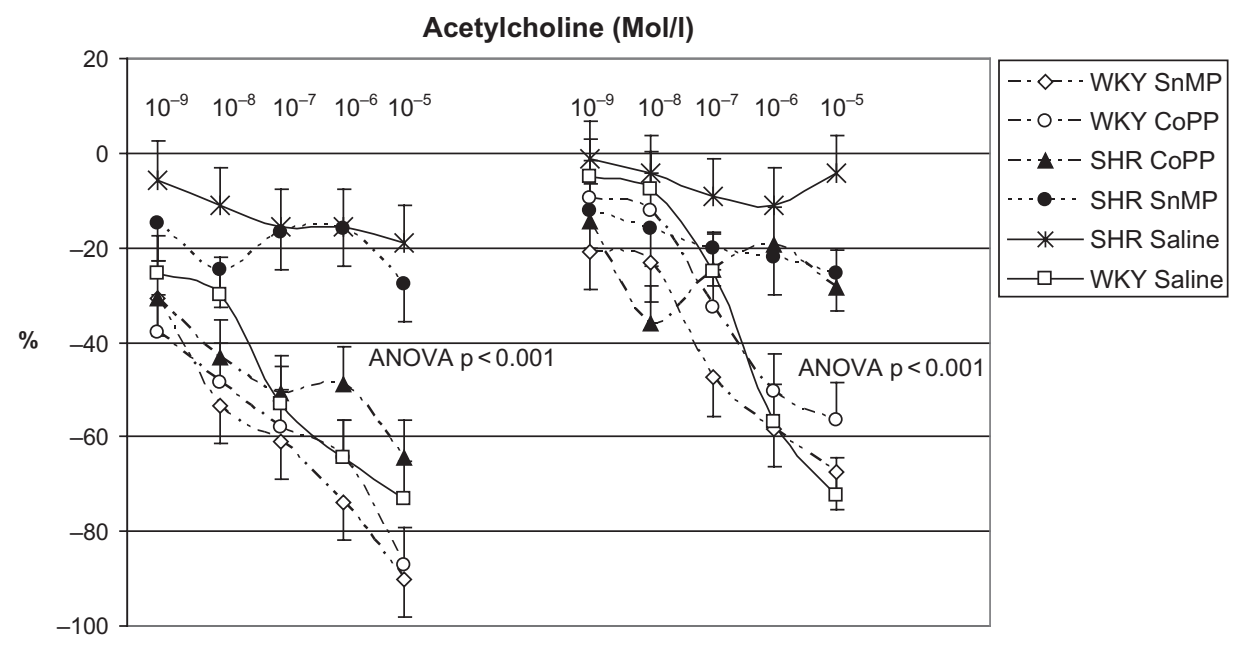

Figure 2. Left panel: concentration-response curve to acetylcholine (from $10^{-9}$ to $10^{-5} \mathrm{~mol} / \mathrm{L}$ ): $\%$ relaxation after precontraction with norepinephrine $10^{-5} \mathrm{~mol} / \mathrm{L}$. Right panel: concentration-response curve to acetylcholine (from $10^{-9}$ to $10^{-5} \mathrm{~mol} / \mathrm{L}$ ) after preincubation with L-NAME $300 \mu \mathrm{mol} / \mathrm{L}$. See text for significance between curves. Abbreviations: WKY SnMP-Wistar-Kyoto rats treated with SnMP $(n=6)$. WKY CoPP-Wistar Kyoto rats treated with CoPP $(n=6)$. SHR SnMP-spontaneously hypertensive rats treated with SnMP $(n=6)$. SHR CoPP-spontaneously hypertensive rats treated with CoPP $(n=6)$. SHR Saline-spontaneously hypertensive rats treated with saline $(n=6)$. WKY Saline-Wistar-Kyoto rats treated with saline $(n=6)$.

the endothelial cells (Table 1, Figure 4). The administration of CoPP increased HO-1 immunostaining in the tunica intima, media, and adventitia of WKY and SHR rats (Table 1, Figure 4). The administration of SnMP did not induce changes in the immunohistochemical pattern of HO-1 immunoreactivity both in WKY and SHR, in respect to saline.

\section{Discussion}

Our study demonstrated that endothelium-dependent vasodilatation in mesenteric small resistance arteries from adult SHR was improved after treatment with the HO inducer cobalt protoporphyrin IX. On the contrary, stannous mesoporphyrin, which inhibits HO, did not modify the vessels' response to acetylcholine and bradykinin, probably because it was maximally impaired. Since the inhibitory effect of L-NAME is present when the NO production is maintained or restored, our interpretation is that in SHR treated with CoPP, basal vasodilation to acetylcholine is improved compared to basal saline (Figure 2, left panel), while L-NAME is able to decrease acetylcholine-dependent responses in CoPPtreated SHR, thus suggesting a restored bioavailability of NO.

A normal microvascular function is maintained provided that anatomical integrity of the endothelial cells and normal vasodilator and contractile responses of vascular smooth muscle cell are present. Spontaneously hypertensive rats represent an experimental model of genetic hypertension which shows several pathological aspects similar to those of human hypertension, namely, an increase in the renin-angiotensin system activity, insulin resistance, dyslipidemia, etc. Young SHRs rapidly develop hypertension and endothelial dysfunction, which are clearly established at the age of 12 weeks (10). Our data demonstrated 


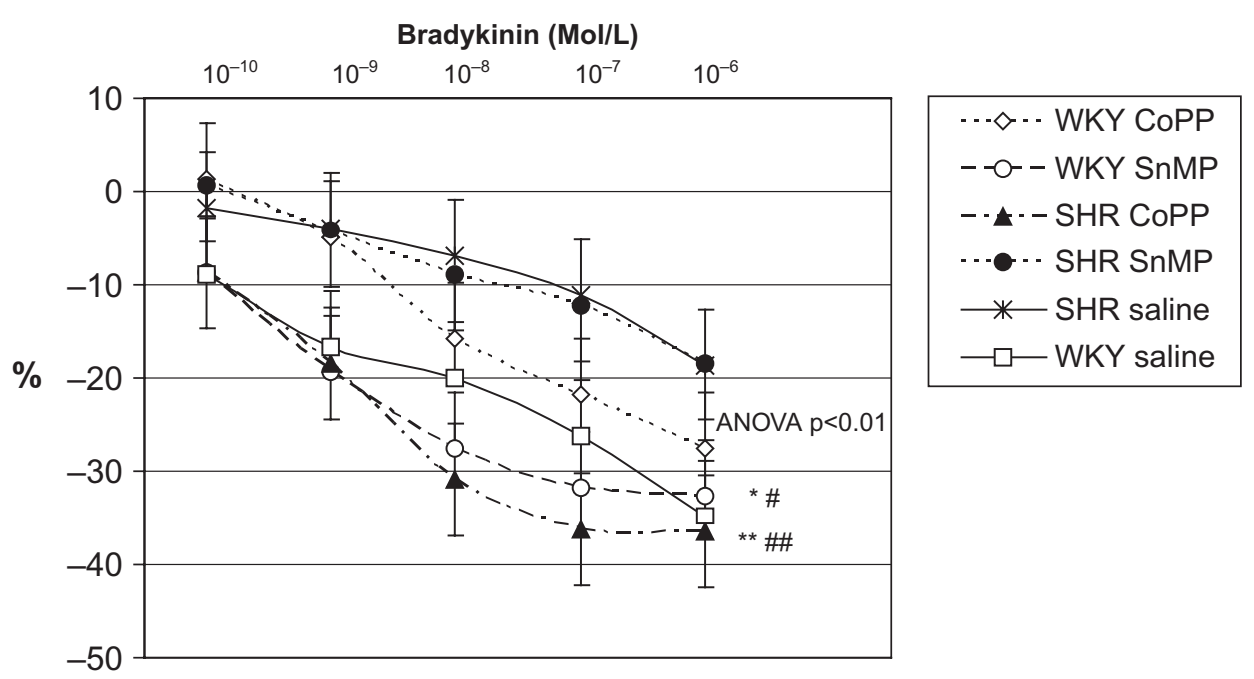

Figure 3. Concentration-response curve to bradykinin (from $10^{-9}$ to $10^{-5} \mathrm{~mol} / \mathrm{L}$ ) of rat mesenteric small arteries: $\%$ relaxation after precontraction with norepinephrine $10^{-5} \mathrm{~mol} / \mathrm{L}$. Abbreviations: WKY SnMP-Wistar-Kyoto rats treated with SnMP $(n=6)$. WKY CoPP-Wistar Kyoto rats treated with CoPP $(n=6)$. SHR SnMP-spontaneously hypertensive rats treated with SnMP $(n=6)$. SHR CoPP-spontaneously hypertensive rats treated with CoPP $(n=6)$. SHR Saline-spontaneously hypertensive rats treated with saline $(n=6)$. WKY Saline-Wistar-Kyoto rats treated with saline $(n=6)$. Saline vs. SHR SnMP.

Table 1

HO-1 immunoreactivity in the mesenteric small resistance arteries of SHR and WKY treated with saline, CoPP, or SnMP

\begin{tabular}{lccc}
\hline & $\begin{array}{c}\text { HO-1 } \\
\text { endothelium }\end{array}$ & $\begin{array}{c}\text { HO-1 tunica } \\
\text { media }\end{array}$ & $\begin{array}{c}\text { HO-1 tunica } \\
\text { adventitia }\end{array}$ \\
\hline SHR Saline $\mathrm{n}=6$ & + & ++ & ++ \\
SHR CoPP $\mathrm{n}=6$ & ++ & +++ & +++ \\
SHR SnMP $\mathrm{n}=6$ & + & ++ & +++ \\
WKY Saline $\mathrm{n}=6$ & ++ & ++ & ++ \\
WKY CoPP $\mathrm{n}=6$ & +++ & +++ & +++ \\
WKY SnMP $\mathrm{n}=6$ & ++ & ++ & ++ \\
\hline
\end{tabular}

Abbreviations: WKY SnMP-Wistar-Kyoto rats treated with SnMP; WKY CoPP-Wistar Kyoto rats treated with CoPP; SHR SnMP-spontaneously hypertensive rats treated with SnMP; SHR CoPP-spontaneously hypertensive rats treated with CoPP; SHR Saline-spontaneously hypertensive rats treated with saline; WKY Saline-Wistar-Kyoto rats treated with saline.

Semi-quantitative data: (-) negative; (+/-) very weak; (+) weak; (++) moderate; $(+++)$ strong.

that a 2 week stimulation of HO-1 is able to induce a significant improvement in endotheliumdependent vasodilatation and this effect is completely abolished by preincubation with the nitric oxide synthase inhibitor L-NMMA. On the other hand, the inhibition of HO-1 activity does not modify the vasodilator response induced by acetylcholine both in SHR and WKY. These results confirm the relevance of the heme/heme oxygenase system in the 

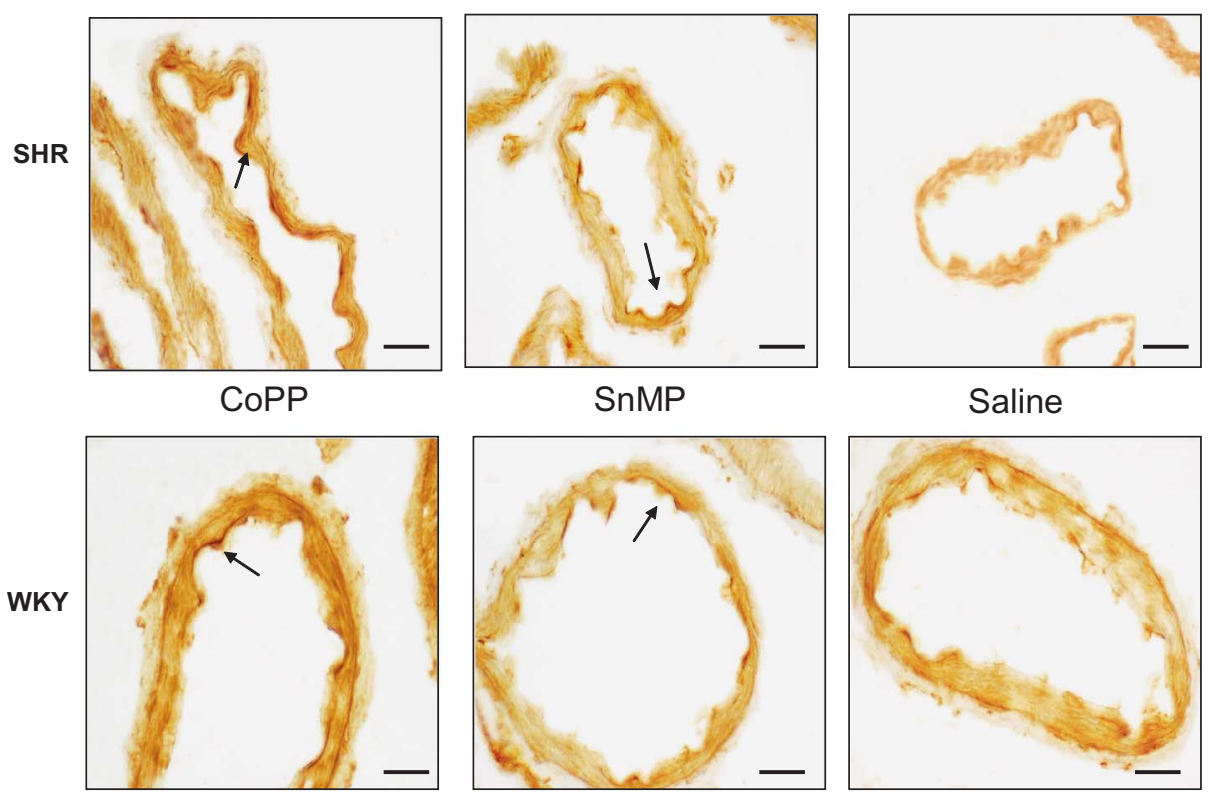

CoPP

SnMP

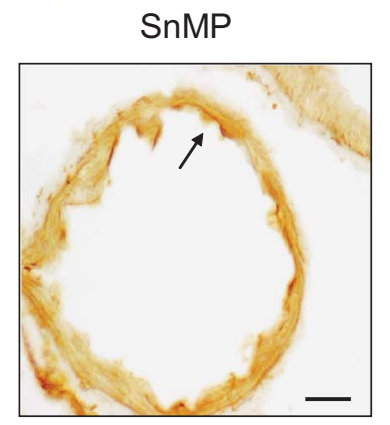

SnMP

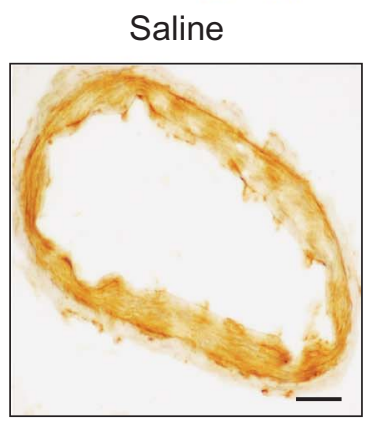

Saline

Figure 4. Immunohistochemical staining of HO-1 of rat mesenteric small arteries. The immunoreactivity appeared as brown spots. Arrows indicate HO-1 immunoreactivity within the endothelial cells. Bar- $20 \mu \mathrm{m}$.

vascular function control and provide an insight into mechanisms that may explain protective effects of HO-1 on hypertensive complications.

It is well known that HO-1 is extremely effective in inhibiting vascular smooth muscle cell growth, and hence, the development of arteriosclerotic processes (16-20). However, the potential clinical application of the stimulation of HO-1 by substances such as cobalt protoporphyrin IX or by gene therapy is still problematic $(21,22)$. In contrast, the products engendered by HO-1 in the degradation of heme, including $\mathrm{CO}$ (23) and biliverdin/bilirubin that mediate the actions of HO-1, seem to be close to a potential application in the clinical setting (24). In fact, biliverdin and bilirubin may inhibit injury-induced vascular smooth muscle cell proliferation and thus prevent narrowing of the vascular lumen in patients with arteriosclerotic lesions associated with chronic graft rejection and balloon injury (23).

Three lines of evidence suggest that bilirubin may have desirable properties. First, bilirubin is a very potent antioxidant that may efficiently scavenge chemically generated peroxyl radicals (25), and therefore, attenuate vascular endothelial activation and dysfunction (26). Second, several studies have shown that individuals with high-normal or slightly above-normal levels of bilirubin have a lesser incidence of atherosclerosis-related diseases than individuals with low-normal levels (27). However, these findings do not necessarily implicate bilirubin as the protective agent given that they are based solely on association studies. Finally, the administration of bilirubin or its precursor biliverdin to rodents can suppress unwanted reactions such as ischemia/reperfusion injury $(23,28)$ and allograft rejection $(23,29)$. However, up until now, few studies have addressed the issue of the role of biliverdin/bilirubin in the prevention of microvascular structural alterations, and in the development of organ damage in hypertension. 
Recently, Wang et al. showed that during a short period of treatment ( 3 weeks), the activation of HO-1 with hemin promotes the regression of vascular structural alterations and normalizes blood pressure values until 9 months after the end of the treatment (30). In a previous study (31), the same authors provided evidence that 8-week SHRs with higher blood pressure values than age-matched WKY have lower basal expression levels of HO-1 and relative transmitters. The dysfunction of the $\mathrm{HO}$ system was already present in a prehypertensive stage and hence seems not a consequence to the development of hypertension. The stimulation by hemin of $\mathrm{HO}$ is able to induce a prevention of the development of hypertension after a short treatment (4 days) started very early, at 7 weeks of age. In fact, 20-week-old SHRs are resistant to HO induction and show no reduction of blood pressure values (31). In our study, we could not test the effect of a longer administration of drugs modulating the HO system, since intraperitoneal administration cannot be extended beyond a couple of weeks. After a short-term treatment with the activator of $\mathrm{HO}$, we could observe a reduction, but not a complete normalization, of blood pressure values. On the contrary, Wang et al. (30) observed a normalization of blood pressure values after 2 weeks of continuous infusion by a subcutaneous hemin-releasing pump; however, in a previous work, the same authors found a reduction (not a normalization) of blood pressure values after intraperitoneal treatment (32). The efficacy of slow release systems could obviously be more effective compared to an intraperitoneal bolus which could have a rather suboptimal peak-trough effect. These aspects may partly explain the different results in terms of blood pressure and vascular morphology between our study and Wang et al's (30). In fact, we could not observe any improvement in structural alterations of small arteries of SHRs treated with CoPP (data not shown). Nevertheless, in our study a significant reduction in SBP was accompanied by a normalization of the endothelial function.

The patho-physiological mechanisms underlying our findings are not clear. Obviously, some of the end-products of HO-1 activation, mainly bilirubin and $\mathrm{CO}$, could exert some beneficial effects on vascular function. HO-1 activators are able to increase bilirubin and biliverdin, which are powerful antioxidants and can thus improve endothelial function by lowering oxidative stress reactions $(26,33)$ and increase the half-life of nitric oxide (8). In our study, the positive effect of CoPP on acetylcholine-induced vasodilatation was abolished by the preincubation with the inhibitor of the inducible nitric oxide synthase L-NMMA. Therefore, it seems probable that an interaction between $\mathrm{CO}$ and nitric oxide is involved in the observed effect: both systems contribute to vascular tone control and play a role in controlling vascular tone. However, we cannot exclude that other modulator processes, closely related to a $\mathrm{HO} / \mathrm{CO}$ system, could be involved in the observed improvement of endothelial function. Heme-oxygenase inducers are also implicated in the cytochrome P-450 dependent arachydonic acid metabolic pathway (34). Since heme constitutes the prosthetic moiety of many cytochrome enzymes by degrading heme, $\mathrm{HO}$ controls the quantity of these enzymes, including cytochrome P-450 monooxygenase.

Finally, a possible limitation of the study is related to the dose of CoPP used in our study. It was previously proposed that some alteration in the cellular CYP340 content may occur, with possible alterations in the tyroid function (35). However, no sign of CoPP toxicity was observed in our study, probably due to the relative short duration of the treatment. A second limitation is the lack of measurements of $\mathrm{HO}$ activity and $\mathrm{HO}$ isoforms protein quantification, considering that immunohistochemistry represent a semi-quantitatve approach. However, our study was aimed at evaluating vascular functional responses, while data about effect of CoPP on HO levels were obtained previously (increased levels of HO-1 mRNA, protein, and activity) (36). 
In our study, SnMP did not worsen endothelium-dependent responses in WKY. Although this finding is partly surprising, it is possible that WKY rats may be resistant to the adverse effects of SnMP, through an activation of controregulatory and protective mechanism. In both SHRs and WKYs, short-term treatment with SnMP induced a reduction of blood pressure. Since the drug passes the blood-brain barrier, it is possible that central mechanisms (reduction in sympathetic nervous system activity or a Adreno Cortico Tropic Hormone (ACTH) release) may be involved in the observed findings (37). The decrease in blood pressure have not influcenced our results in terms of vascular functional responses, since in our study they have been evaluated in vitro, with no actual pressure or flow. Finally, no difference in HO immunoreactivity between SHRs and WKYs was observed in our study, probably due to the relative lack of sensitivity of our semi-quantitative approach.

In conclusion, as previously mentioned, the $\mathrm{HO} / \mathrm{CO}$ metabolic pathway is believed to be involved in the regulation of basal tone of resistance blood vessels and also in the development of vascular remodeling. Modulation of HO by appropriate drugs may improve endothelium-dependent dilatation of small arteries, reduce blood pressure and oxidative stress in animal models, and may also delay the progression of human atherosclerotic lesions. Therefore, possible clinical application of pharmacological modulation of an HO system is of primary interest and importance. Our data provide a contribution in this regard.

Therefore, our results indicate that the stimulation of $\mathrm{HO}$ seems to induce an improvement of endothelial function in SHRs, possibly by reducing oxidative stress and increasing NO availability. Further studies are needed to precisely elucidate the underlying mechanism, as well as the potential clinical application of drugs modulating HO.

\section{Acknowledgments}

The authors thank Annamaria Pilu for technical contribution and Professor Asar Lucchini for her linguistic revision.

\section{Declaration of Interest}

The authors report no conflicts of interest. The authors alone are responsible for the content and writing of the paper.

\section{References}

1. Brunner H, Cockcroft JR, Deanfield J, Donald A, Ferrannini E, Halcox J, Kiowski W, Luscher TF, Mancia G, Natali A, Oliver JJ, Pessina AC, Rizzoni D, Rossi GP, Salvetti A, Spieker LE, Taddei S, Webb DJ, the Working Group on Endothelins and Endothelial Factors of the European Society of Hypertension. Endothelial function and dysfunction. Part II: Association with cardiovascular risk factors and diseases. A statement by the Working Group on Endothelins and Endothelial Factors of the European Society of Hypertension. J Hypertens 2005;23:233-246.

2. Drexler H. Endothelial dysfunction: Clinical implications. Prog Cardiovasc Dis 1997; 39:287-324.

3. Taddei S, Salvetti A. Endothelial dysfunction in essential hypertension: Clinical implications. $J$ Hypertens 2002; 20:1671-1674.

4. Morita T. Heme oxygenase and atherosclerosis. Arterioscler Thromb Vasc Biol 2005; 25:1786-1795.

5. Mulvany MJ, Aalkjaer C. Structure and function of small arteries. Physiol Rev 1990; 70:921-961.

6. Deng LY, Li JS, Schiffrin EL. Endothelium-dependent relaxation of small arteries from essential hypertensive patients: Mechanisms and comparison with normotensive subjects and with responses of vessels from spontaneous hypertensive rats. Clin Sci 1995; 88:611-622. 
7. Rizzoni D, Porteri E, Castellano M, Bettoni G, Muiesan ML, Tiberio G, Giulini SM, Rossi GP, Bernini G, Agabiti Rosei E. Endothelial dysfunction in hypertension is independent from the etiology and from vascular structure. Hypertension 1998; 31(part 2):335-341.

8. Wu L, Wang R. CO monoxide: Endogenous production, physiological functions, and pharmacological applications. Pharmacol. Rev 2005; 57:585-630.

9. Mulvany MJ, Aalkjaer C. Structure and function of small arteries. Physiol Rev 1990; 70:921-971.

10. Rizzoni D, Castellano M, Porteri E, Bettoni G, Muiesan ML, Agabiti-Rosei E. Vascular structural and functional alterations before and after the development of hypertension in SHR. Am J Hypertens 1994; 7:193-200.

11. Mulvany MJ, Hansen PK, Aalkjaer C. Direct evidence that the greater contractility of resistance vessels in spontaneously hypertensive rats is associated with a narrowed lumen, a thickened media, and an increased number of smooth muscle cell layers. Circ Res 1978; 43:854-864.

12. Rizzoni D, Castellano M, Porteri E, Bettoni G, Muiesan ML, Agabiti Rosei E. Delayed development of hypertension after short-term nitrendipine treatment. Hypertension 1994; 24:131-139.

13. Vecchione C, Frata L, Rizzoni D, Notte A, Poulet R, Porteri E, Frati G, Guelfi D, Trimarco V, Mulvany MJ, Agabiti Rosei E, Trimarco B, Cotecchia S, Lembo G. Cardiovascular influences of $\alpha_{1 \mathrm{~B}}$-adrenergic receptor in mice. Circulation 2002; 105:1700-1707.

14. Brunner H, Cockcroft JR, Deanfield J, Donald A, Ferrannini E, Halcox J, Kiowski W, Lüscher TF, Mancia G, Natali A, Oliver JJ, Pessina AC, Rizzoni D, Rossi GP, Salvetti A, Spieker LE, Taddei S, Webb DJ, the Working Group on Endothelins and Endothelial Factors of the European Society of Hypertension. Endothelial function and dysfunction. Part II: Association with cardiovascular risk factors and diseases. A statement by the Working Group on Endothelins and Endothelial Factors of the European Society of Hypertension. J Hypertens 2005, 23:233-246.

15. Rizzoni D, Porteri E, Guelfi D, Muiesan ML, Valentini U, Cimino A, Girelli A, Rodella L, Bianchi R, Sleiman I, Agabiti Rosei E. Structural alterations in subcutaneous small arteries of normotensive and hypertensive patients with non-insulin-dependent diabetes mellitus. Circulation 2001; 103:1238-1244.

16. Yet SF, Layne MD, Liu X, Chen YH, Ith B, Sibinga NE, Perrella MA. Absence of heme oxygenase-1 exacerbates atherosclerotic lesion formation and vascular remodeling. FASEB $J$ 2003;17: $1759-1761$.

17. Durante W. Heme oxygenase-1 in growth control and its clinical application to vascular disease. J Cell Physiol 2003;195:373-382.

18. Duckers HJ, Boehm M, True AL, Yet SF, San H, Park JL, Clinton Webb R, Lee ME, Nabel GJ, Nabel EG. Heme oxygenase-1 protects against vascular constriction and proliferation. Nat Med 2001; 7:693-698.

19. Liu XM, Chapman GB, Wang H, Durante W. Adenovirus-mediated heme oxygenase-1 gene expression stimulates apoptosis in vascular smooth muscle cells. Circulation 2002; 105:79-84.

20. Öllinger R, Bilban M, Erat A, Froio A, McDaid J, Tyagi S, Csizmadia E, Graça-Souza AV, Liloia A. Soares MP, Otterbein LE, Usheva A, Yamashita K, Bach FH. Bilirubin A: Natural inhibitor of vascular smooth muscle cell proliferation. Circulation 2005;112:1030-1039.

21. Barceloux DG. Cobalt. J Toxicol Clin Toxicol 1999;37:201-206.

22. Pislaru S, Janssens SP, Gersh BJ, Simari RD. Defining gene transfer before expecting gene therapy: Putting the horse before the cart. Circulation 2002; 106:631-636.

23. Otterbein LE, Zuckerbraun BS, Haga M, Liu F, Song R, Usheva A, Stachulak C, Bodyak N, Smith RN, Csizmadia E, Tyagi S, Akamatsu Y, Flavell RJ, Billiar TR, Tzeng E, Bach FH, Choi AM, Soares MP. CO monoxide suppresses arteriosclerotic lesions associated with chronic graft rejection and with balloon injury. Nat Med 2003; 9:183-190.

24. Abraham NG, Kappas A. Pharmacological and clinical aspects of heme oxygenase. Pharmacol Rev 2008; 60:79-127.

25. Stocker R, Yamamoto Y, McDonagh AF, Glazer AN, Ames BN. Bilirubin is an antioxidant of possible physiological importance. Science 1987; 235:1043-1046. 
26. Kawamura K, Ishikawa K, Wada Y, Kimura S, Matsumoto H, Kohro T, Itabe H, Kodama T, Maruyama Y. Bilirubin from heme oxygenase-1 attenuates vascular endothelial activation and dysfunction. Arterioscler Thromb Vasc Biol 2005; 25:155-160.

27. Novotny L, Vitek L. Inverse relationship between serum bilirubin and atherosclerosis in men: a meta-analysis of published studies. Exp Biol Med 2003; 228:568-571.

28. L'Abbate A, Neglia D, Vecoli C, Novelli M, Ottaviano V, Baldi S, Barsacchi R, Paolicchi A, Masiello P, Drummond GS, McClung JA, Abraham NG. Beneficial effect of heme oxygenase-1 expression on myocardial ischemia-reperfusion involves an increase in adiponectin in mildly diabetic rats. Am J Physiol Heart Circ Physiol 2007; 293:H3532-H3541.

29. Ollinger R, Wang H, Yamashita K, Wegiel B, Thomas M, Margreiter R, Bach FH. Therapeutic applications of bilirubin and biliverdin in transplantation. Antioxid Redox Signal 2007; 9:2175-2185.

30. Wang R, Shamloul R, Wang X, Meng Q, Wu L. Sustained normalization of high blood pressure in spontaneously hypertensive rats by implanted hemin pump. Hypertension 2006; 48:685-692.

31. Ndisang JF, Zhao W, Wang R. Selective regulation of blood pressure by heme oxygenase- 1 in hypertension. Hypertension 2002; 40;315-321.

32. Wang R, Ndisang JF, Wang X, Shamloul R, Wu L. Unprecedented long-lasting normalization of hypertension by a hemin therapy. Circulation 2005;112:II-511 (Abstract).

33. Stasch JP, Schmidt PM, Nedvetsky PI, Nedvetskaya TY, Kumar AHS, Meurer S, Deile M, Taye A, Knorr A, Lapp A Müller H, Turgay Y, Rothkegel C, Tersteegen A, Kemp-Harper B. MüllerEsterl W, Schmidt HHHW. Targeting the heme-oxidized nitric oxide receptor for selective vasodilatation of diseased blood vessels. J Clin Invest 2006; 116:2552-2561.

34. Kumar S, Bandyopadhyay U. Free heme toxicity and its detoxification systems in human. Toxicology Letters 2005; 157:175-188.

35. Galbraith RA, Kappas A. Regulation of food intake and body weight in rats by the synthetic heme analogue cobalt protoporphyrin. Am J Physiol 1991; 261(6 Pt 2):R1388-1394.

36. von Dobschuetz E, Schmidt R, Scholtes M, Thomusch O, Schwer CI, Geiger KK, Hopt UT, Pannen BH. Protective role of heme oxygenase-1 in pancreatic microcirculatory dysfunction after ischemia/reperfusion in rats. Pancreas 2008; 36:377-384.

37. Turnbull AV, Kim CK, Lee S, Rivier CL. Influence of carbon monoxide, and its interaction with nitric oxide, on the adrenocorticotropin hormone response of the normal rat to a physicoemotional stress. J Neuroendocrinol 1998; 10:793-802. 